\title{
A Data Fusion Algorithm for Multi-Sensor Microburst Hazard Assessment
}

\author{
Craig R. Wanke* and R. John Hansman ** \\ Department of Aeronautics and Astronautics \\ Massachusetts Institute of Technology \\ Cambridge, MA USA
}

\begin{abstract}
A recursive model-based data fusion algorithm for multi-sensor microburst hazard assessment is described. An analytical microburst model is used to approximate the actual windfield, and a set of "best" model parameters are estimated from measured winds. The winds corresponding to the best parameter set can then be used to compute alerting factors such as microburst position, extent, and intensity. The estimation algorithm is based on an iterated extended Kalman filter which uses the microburst model parameters as state variables. Microburst state dynamic and process noise parameters are chosen based on measured microburst statistics. The estimation method is applied to data from a time-varying computational simulation of a historical microburst event to demonstrate its capabilities and limitations. Selection of filter parameters and initial conditions is discussed.

Computational requirements and datalink bandwidth considerations are also addressed.
\end{abstract}

\section{Introduction}

Low altitude wind shear has been a major cause of fatal aviation accidents in the U.S. 1 The localized intense downdrafts known as microbursts are the most dangerous form of wind shear, and pose a serious hazard to aircraft during takeoff or approach. In a typical microburst encounter, an aircraft first encounters a performanceincreasing headwind. This is followed by a downdraft and a rapid transition from headwind to tailwind, which produce sharp losses in altitude and/or airspeed.

Several systems for detection and measurement of microburst hazards are currently nearing the operational stage. Effective ground-based systems such as Terminal Doppler Weather Radar (TDWR) and the extended Low Level Wind Shear Alert System (LLWAS) are entering the deployment phase. TDWRs will be located at 47 major airports, and detect microbursts primarily by measuring the surface wind velocity component radial to the radar and identifying areas of radial shear. ${ }^{2,3}$ LLWAS is a network of anemometers which measure horizontal windspeed and direction around the airport surface, and detect wind shear events from differences in wind speed and direction between sensors. ${ }^{4}$ Airbome reactive wind shear alerting systems, currently in use, detect microburst penetration by

\footnotetext{
* Research Assistant

** Associate Professor, Associate Fellow AIAA Copyright (O) 1992 by M.I.T. Published by the American Institute of Aeronautics and Astronautics, Inc. with permission.
}

comparing inertial and air data system measurements to compule the local winds. Several types of airborne forward-look sensor technologies are also under development. including infrared radiometry. Doppler radars and Doppler lidars. 5 Infrared systems measure the drop in temperature associated with the air in the center of a microburst, while Doppler radars and lidars measure wind velocities along the flight path ahead of the aircraft. In addition to new sensor developments, the development of digital air-ground datalink capabilities such as the Mode-S beacon system will allow microburst alert information to be exchanged between air and ground-based systems (Figure 1).6

As new detection systems become operational, it will become likely that more than one sensor system will be available in a given situation. Also, each of the aforementioned sensor systems has some geometrical observability problems. For example, both ground-based and airborne Doppler radars and lidars can only measure wind velocities radial to the sensor, not vertical winds. The aviation hazard posed by a microburst, however, is due to both horizontal wind shear and downdrafts in the microburst core. Therefore, a technique for combining data from different systems with different measurement characteristics could improve estimates of microburst hazards and aid alert generation.

The goal of this "data fusion" process is to provide a microburst detection and hazard assessment capability which is significantly better than that which can be achieved using a single sensor. The data fusion algorithm must provide appropriale information for alert generation, in a timely fashion, and be feasible with regard to the available air-ground datalink bandwidth and computational capabilities. Previous work at MIT has focused on

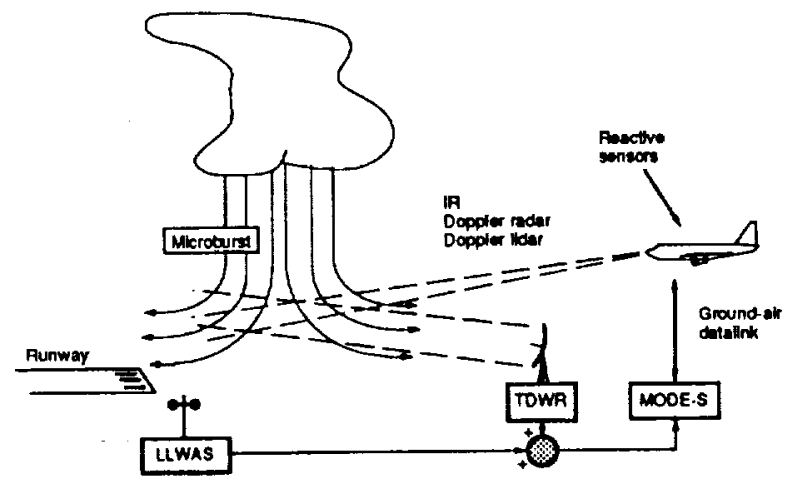

Figure 1: Advanced Microburst Detection and Alerting Systems

Research supported by government grant. 
definition of appropriate information for microburst alerts. This work has included analytical studies to determine appropriate microburst hazard criteria, ${ }^{7}$ and piloted parttask simulator studies to determine the information requirements of the flight crew when faced with a microburst alert situation. 8,9 The conclusions of these studies were that estimates of microburst location, extent, and approximate intensity are required for alerting purposes, and that microburst intensity can be quantified well using criteria which relate to the expected aircraft energy loss due to the microburst windfield. Therefore, a good data fusion algorithm should be able to compute the position and extent of a microburst, and to estimate intensity including contributions from both horizontal wind shear and downdrafts.

The data-fusion process can be done on a number of levels. One approach is to merge the final products of the sensor systems to produce improved alerts. For example, product-level techniques have been used to integrate TDWR and LLWAS information 10 and to determine the probability of hazardous wind shear given a wide range of evidence. II Another approach is to integrate sensors on the data level. The data-level approach is more complex, due to the large volume of data produced by several wind shear sensor systems. However, if correctly implemented, observability problems due to poor sensor geometry can be alleviated. Data from multiple sensors can be combined to form a "super sensor" which has improved sensing geometry. The technique proposed in this paper is a model-based data-level approach which attempts to gain this observability benefit without prohibitively large computational or data transmission requirements.

\section{Model-Based_Approach}

\section{Why Use a Model?}

Representation of the actual microburst windficld with an analytical model has two major advantages. Firstly, once the model has been "fitted" to the windfield, the windfield can be approximated by the values of the model parameters. Thus, if the model represents the windfield well enough, the measured information (a large data set) can be encapsulated in a small set of "best-fit" model parameters. Since it is impractical (or at least undesirable) to transmit raw data between aircraft and ground-based systems, this is an important advantage.

Secondly, an analytical model can include additional information which can be used to infer quantities which cannot be directly measured, such as inferring vertical velocities from radar-measured radial velocities. Analytical models can be designed to satisfy basic fluid dynamic relationships such as mass continuity, and can be adjusted to reflect results of microburst field measurements.

\section{Analytical Microburst Model}

The analytical microburst model used in this work was developed at NASA Langley Research Center initially by Oseguera and Bowles. ${ }^{12}$ and later improved by Vicroy. 13 The Oseguera-Bowles-Vicroy (OBV) model uses shaping functions to gencrale an axisymmetric flowfield which satisfies the mass continuity equation and is representative of the major characteristics of measured microbursts. Sample winds for a constant-altitude path through the model windfield are shown in Figure 2. The horizontal winds exhibit the classic microburst characteristic of a headwind increase, followed by rapid shearing to a tailwind. The vertical wind plot shows a downdraft in the microburst center and smaller updrafts at the edges.

The microburst winds are uniquely defined by a set of five parameters and three empirically-adjusted constants. For this study, a simple ambient wind ( 4 additional parameters) was added to the microburst windfield. The model parameters are summarized in Table 1 . The total winds are given by non-linear, smooth, differentiable functions of the parameters and a given $(x, y, h)$ position as follows:

$$
\begin{aligned}
& \mathrm{U}=\mathrm{U}_{\text {microburst }}+\mathrm{U}_{0}+\mathrm{U}_{\mathrm{h}} \mathrm{h} \\
& \mathrm{V}=\mathrm{V}_{\text {microburst }}+\mathrm{V}_{0}+\mathrm{V}_{\mathrm{h}} \mathrm{h} \\
& \mathrm{W}=\mathrm{W}_{\text {microburst }}
\end{aligned}
$$

$U, V$, and $W$ are the Eastward, Northward, and vertical wind velocities; $h$ is the altitude above ground level. "Microburst" quantities are functions of position and of the first five parameters in Table 1; these functions are summarized in Appendix A.

The OBV model is axisymmetric, but naturally occurring microbursts are often asymmetric. ${ }^{14}$ In addition, multiple microbursts have been observed to occur close together and interact. To handle these cases, the model was extended to allow multiple interacting microbursts. For each microburst, another set of five microburst model parameters (the first five in Table 1) can be added. It is assumed that the ambient wind will be

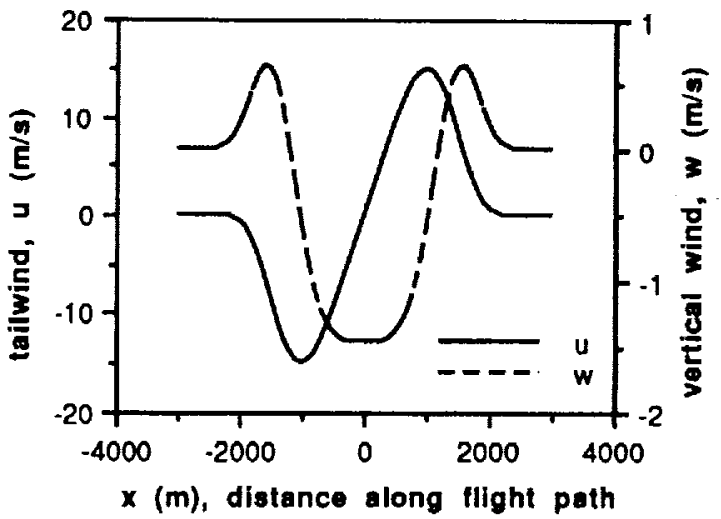

Figure 2: Microburst Model Windfield. Sample winds for a constant-altitude flight path through the center of a simulated microburst. 
Table 1: Modified Oseguera-Bowles-Vicroy Microburst Model Parameters

\begin{tabular}{|l|l|}
\hline Parameter & \multicolumn{1}{|c|}{ Description } \\
\hline $\mathrm{x}_{0}$ & X-coordinate (East) of inicroburst center $(\mathrm{m})$ \\
\hline $\mathrm{y}_{0}$ & Y-coordinate (North) of microburst center $(\mathrm{m})$ \\
\hline$U_{\mathrm{m}}$ & Maximum horizontal outflow speed $(\mathrm{m} / \mathrm{s})$ \\
\hline $\mathrm{R}_{\mathrm{p}}$ & Radius of maximum outflow (meters) \\
\hline $\mathrm{Z}_{\mathrm{m}}$ & Altitude AGL of maximum outflow (meters) \\
\hline$U_{0}$ & Eastward ambient wind constant component $(\mathrm{m} / \mathrm{s})$ \\
\hline$U_{\mathrm{h}}$ & Eastward ambient wind altitude gradient $(\mathrm{m} / \mathrm{s} / \mathrm{m})$ \\
\hline$V_{0}$ & Northward ambient wind const. component $(\mathrm{m} / \mathrm{s})$ \\
\hline$V_{h}$ & Northward ambient wind altitude gradient $(\mathrm{m} / \mathrm{s} / \mathrm{m})$ \\
\hline
\end{tabular}

roughly constant throughout the $x-y$ space of interest, i.e. near the airport, and so only one set of ambient wind parameters is used. The winds from each model microburst are summed to get the overall model windfield; this superposition does not violate mass continuity. In the simulation study below, when a "two-microburst" model is referred to it does not necessarily indicate that there are two microbursts being detected, but that two superimposed model microbursts are being used to simulate a complex microburst windfield with more than one area of high downdraft speed.

\section{Model-Based Multi-Sensor Data Fusion}

Given a suitable model, the fusion problem reduces to estimation of the "best" set of model parameters based on all available wind measurements. Once the best Darameters have been estimated, alerting factors (intensity. extent etc.) can be derived from the analytical model windfield corresponding to the estimated model Darameters.

This estimation procedure must satisfy several constraints to be practical. The estimation algorithm must be recursive, to handle new measurements as they become available. It must also account for time variation in the model parameters, since microbursts are dynamic phenomena with short lifetimes on the order of 15 minutes and sensor measurements will be taken at different times. It should also be probabilistic, to take advantage of microburst statistical characteristics from past field studies. A Kalman filter approach is proposed to satisfy these requirements.

\section{Iterated Extended Kalman Filter Algorithm}

\section{Estimation Problem Structure}

Kalman filtering techniques require a state-space dynamic model of the system and a relationship between system parameters and measured quantities. In this case, we would like to estimate analytical model parameters which best describe the microburst from measurements of the winds. The analytical microburst model parameters are therefore used as the filter state variables $x(t)$. It was assumed that the time evolution of the microburst parameters can be adequately modeled by a linear, timeinvariant, continuous-time system:

$$
\dot{x}(1)=A x(t)+B u(t)+L w(1)
$$

Deterministic inputs to the system are represented by $u(t)$, and $w(t)$ is a white Gaussian process noise input. The A. B. and L matrices define the dynamic model; they will be discussed below. Since the state variables $x(t)$ are the analytical model parameters, they are related to the wind measurements through the analytical model wind equations. The resulting non-linear discrete-time measurement equation is:

$$
z_{k}=h_{k}\left(x\left(t_{k}\right)\right)+v_{k}
$$

where the measurement equations $h_{k}$ are simply the wind equations from the analytical model, and $v_{k}$ represents measurement noise. The state vector, $x$, and error covariance matrix, P, for a single downdraft case are defined as follows:

$$
\begin{aligned}
& x=\left[\begin{array}{lllllllll}
x_{0} & y_{0} & U_{m} & R_{p} & Z_{m} & U_{0} & U_{h} & V_{0} & V_{h}
\end{array}\right]^{T} \\
& P=E\left[\begin{array}{lll}
(x-\hat{x})(x-\hat{x})^{T}
\end{array}\right]
\end{aligned}
$$

where $\hat{x}$ is the current parameter estimate. The microburst eastward core location, $x_{0}$, should not be confused with $x$, the state vector. Process noise, $w(t)$, and measurement noise, $v_{k}$, are white and gaussian with the following characteristics:

$$
\begin{aligned}
& E\left[(L w(t))(L w(\tau))^{T}\right]=L Q(t) L^{T} \delta(t-\tau) \\
& E\left[v_{k} v_{k}^{T}\right]=R_{k}
\end{aligned}
$$

The aim of the filter is to produce the state estimate $\hat{x}$ which minimizes the error covariance $P$. Since the measurement equation is non-linear, this cannot be done with a standard Kalman filter algorithm. An extended Kalman filter (EKF) approach was chosen. The structure and principal equations for the EKF are briefly described below, based on the formulation given in Ref. 15. The filtering algorithm for discrete-time measurements is a two step process: (1) apply the system dynamic model to propagate the state estimate and state estimation error covariance between measurements, and (2) update the estimate when new measurements arrive.

Estimate Propagation: Microburst Dynamic Modeling

For linear, time-invariant, continuous-time system dynamics the propagation of the state estimate and estimation error covariance between measurements is governed by:

$$
\dot{\hat{x}}(t)=A \hat{x}(t)+B u(t)
$$




$$
\dot{P}(t)=A P(t)+P(t) A^{T}+L Q(1) L^{T}
$$

The A, B, L, and Q matrices define the microburst time-evolution dynamics. Since the analytical model is lime-invariant, these parameters must come from another source. Unfortunately, there is no simple time-varying analytical model available. However, measured microburst statistics can be used to approximate some dynamics. For example, microburst radial extent tends to increase steadily throughout the microburst lifecycle. Analysis of data from Colorado microburst measurements 16.17 indicates that the change in radial extent vs. time can be approximated by a constant bias (a) with additive white zero-mean gaussian noise (n):

$$
\dot{\mathrm{R}}_{\mathrm{p}}=\mathrm{a}+\mathrm{n}(\mathrm{t})
$$

where $\mathrm{a}=0.102 \mathrm{~km} / \mathrm{min}$ and the noise term has a standard deviation of $0.15 \mathrm{~km} / \mathrm{min}$. The constant bias is treated as a deterministic input, and the noise term leads to a value for one element in the $Q$ matrix. Similar modeling may be possible for some of the other state variables. For example, motion of the microburst core $\left(\dot{x}_{0}, \dot{y}_{0}\right)$ may be related to the ambient wind parameters, which would lead to non-zero entries in the A matrix. In the simulations discussed below, the A matrix was assumed to contain all zeros. The $B$ and $Q$ matrix elements were set based on statistical information where possible, and from engineering judgement when no statistical information was available. Further research on microburst dynamics is currently in progress.

Since the time behavior of the microburst parameters is not well modeled, significant process noise is required. The use of process noise to compensate for modeling deficiencies is similar to the well-known technique of applying a "forgetting factor" to older data in a baich leastsquares formulation. In any case, these simple dynamics lead to sparse A, B, L, and Q matrices, and the propagation step in the filter requires little computation.

\section{Incorporating Measurements}

When new measurements are taken, the estimate is updated. The non-linear measurement equation, however, makes the update process difficult. The formulation presented here is based on the extended Kalman filter update with the addition of a local iteration procedure to reduce the effects of the measurement non-linearities. 15,18 At time $t_{k}$, a local iteration (over $i$ ) is performed. The $i^{\text {th }}$ parameter estimate at time $t_{k}, \hat{x}_{k, j}$, is updated with the following expression:

$$
\begin{aligned}
& \hat{x}_{k, j+1}^{+}=\hat{x}_{k}^{-}+K_{k, i}\left[z_{k}-h_{k}\left(\hat{x}_{k, j}^{+}\right)-H_{k}\left(\hat{x}_{k, i}^{+}\right)\left(\hat{x}_{k}^{-}-\hat{x}_{k, j}^{+}\right)\right] \\
& \hat{x}_{k, 0} \equiv \hat{x}_{k}, \quad i=0,1, \ldots
\end{aligned}
$$

The Kalman gain, $\mathrm{K}$, is ordinarily computed from:

$$
K_{k, i}=P_{k} H_{k}^{T}\left(\hat{x}_{k, i}^{+}\right)\left[H_{k}\left(\hat{x}_{k, i}^{+}\right) P_{k}^{-} H_{k}^{T}\left(\hat{x}_{k, i}^{+}\right)+R_{k}\right]^{-1}
$$

and $H_{k}$ is the locally linearized measurement matrix:

$$
H_{k}(\hat{x})=\left[\frac{\partial h_{k}(x)}{\partial x}\right]_{x=\hat{x}}
$$

In the above expressions, $\hat{x}_{k}$ and $P_{k}$ indicate the propagated estimate and error covariance al time $t_{k}$ (prior to updating), while $\hat{x}_{k}^{+}$and $P_{k}^{+}$indicate the updated estimate and covariance based on the measurement $z_{k}$. The local iteration is repeated until the scaled norm of the parameter estimate does nol change significantly. After the new estimate has been produced, the updated error covariance matrix is computed using values from the final iteration step:

$$
P_{k}^{+}=\left[I-K_{k, j} H_{k}\left(\hat{x}_{k, i}^{+}\right)\right] P_{k}^{-}
$$

Some simple testing, in which winds generated directly from the OBV model were "identified" using this algorithm, indicated that the iterated filter results in significantly better estimates than the standard EKF; this has also been found by other investigators. ${ }^{19} \mathrm{~A}$ probabilistic interpretation of this iteration based on Bayesian maximum likelihood estimation is given in Ref. 18.

One difficulty with the above updating algorithm is that there may be large numbers of measurements available at a single time step (as in TDWR data, for example), and the computation of the Kalman gain (Eqn. 15) requires inversion of an $r$-by-r matrix, where $r$ is the number of measurements. The number of computations required to do this scales as $\mathrm{r}^{3}$. In a linear filter, a large batch of measurements can be treated as a series of sequential scalar measurements (occurring at infinitesmal time spacing) without loss of information, thereby avoiding this problem. When the measurement equation is non-linear, the measurements cannot be incorporated sequentially without losing a significant amount of information. Therefore, an alternate form of the gain computation is required. When the number of measurements exceeds twice the number of states, and the measurement noises are independent (diagonal $R_{k}$ ) it is more efficient to use the "information form" of the gain computation:

$$
\begin{aligned}
& \left(\mathrm{P}_{k}^{-1}\right)^{+}=\left(\mathrm{P}_{k}^{-1}\right)+\mathrm{H}_{k}^{\mathrm{T}} \mathrm{R}_{k}^{-1} \mathrm{H}_{k} \\
& \mathrm{~K}_{k}=\left(\mathrm{P}_{k}^{-1}\right)^{+} \mathbf{H}_{k}^{\mathrm{T}} \mathbf{R}_{k}^{-1}
\end{aligned}
$$

This form can be readily applied to the iterated EKF update described above. Although the covariance update must now be done inside the loop, the required matrix inversion is only $n-b y-n$, where $n$ is the number of states (model parameters). The computational requirement now scales linearly with $r$ and cubically with $n$. In the 
simulation cases below, where $r>100$ and $n=9$ or 14 . this form was found to be much more efficient.

\section{Multiple-Microburst Form}

As discussed above, several model microbursts can be superimposed to simulate a more complex windfield. In this case. 5 new states are added for each additional model microburst. For $i$ microbursts, the full state vector, $x$, is defined as:

$$
\begin{aligned}
& x=\left[\begin{array}{llllllll}
x_{m b, l}^{T} & x_{m b, 2}^{\top} & \ldots & x_{m b, i}^{T} & U_{0} & U_{h} & V_{0} & V_{h}
\end{array}\right]^{T} \\
& \text { where: } \\
& x_{m b, i}=\left[\begin{array}{lllllll}
x_{0, i} & y_{0, i} & U_{m, i} & R_{p, i} & Z_{m, i}
\end{array}\right]^{\top}
\end{aligned}
$$

In the simulations discussed below, one- and twomicroburst forms are used.

\section{Initialization}

This algorithm can incorporate multi-sensor data, given that the microburst has previously been detected. The assumption is made that a single sensor has detected the event and has produced an initial parameter estimate and associated error covariance. The initialization algorithm therefore depends on the measurement characteristics of the initial sensor. The general process, however, is the same for all sensors. Quantities that can be directly measured are estimated from the initial data set, and quantities which are unobservable are initialized using statistics derived from microburst field studies.

For example, if a TDWR initially detects a microburst, estimates of maximum outflow speed $\left(U_{m}\right)$, outflow radius $\left(R_{p}\right)$, and core position $\left(x_{0} . y_{0}\right)$ can be derived from the radial flowfield measured by TDWR. The outflow depth $Z_{m}$ is an unobservable parameter, and must be initialized from statistics. Outflow depth statistics have been measured for 26 Colorado microbursts, 20 and the mean altitude of maximum outflow velocity was found to be 109 meters. This value was used to initialize the filter for the simulations discussed below; in which TDWR was always assumed to make the initial detection. The initial covariance matrix was diagonal, and values were chosen based on sensor resolution criteria or statistics where possible.

\section{Simulation Results}

\section{Figures of Merit}

As mentioned above, the important quantities for alerting purposes are position, extent, and approximate intensity. The "effectiveness" of the proposed algorithm can be defined in terms of its capability to produce these quantities. Therefore, iwo figures of merit were defined. The first concerns position and extent. Given a center point, an "extent polygon" can be drawn for a microburst windfield (example shown in Figure 3). ${ }^{14}$ The vertices of the polygon correspond to the points of maximum radial outflow speed (measured radially outward from a center point). This polygon encloses the entire performancedecreasing portion of the microburst. The ability of the model-based algorithm to define this hazardous region can then be evaluated by comparing the extent polygon $A$ of the truth windfield to the extent polygon $B$ of the analytical model windfield corresponding to the estimated parameters:

$$
M_{e x t e n t}=\frac{A \cap B}{A \cup B}
$$

This quantity has a maximum value of $I$ (for an exact match) and falls off for both underestimation and overestimation of the extent boundaries (Figure 4). Core position errors are also reflected, since the model extent polygon is then laterally translated with respect to the true extent polygon. This quantity is a function of altitude. but the dependence was found to be very weak and only results for a single altitude are presented in this paper.

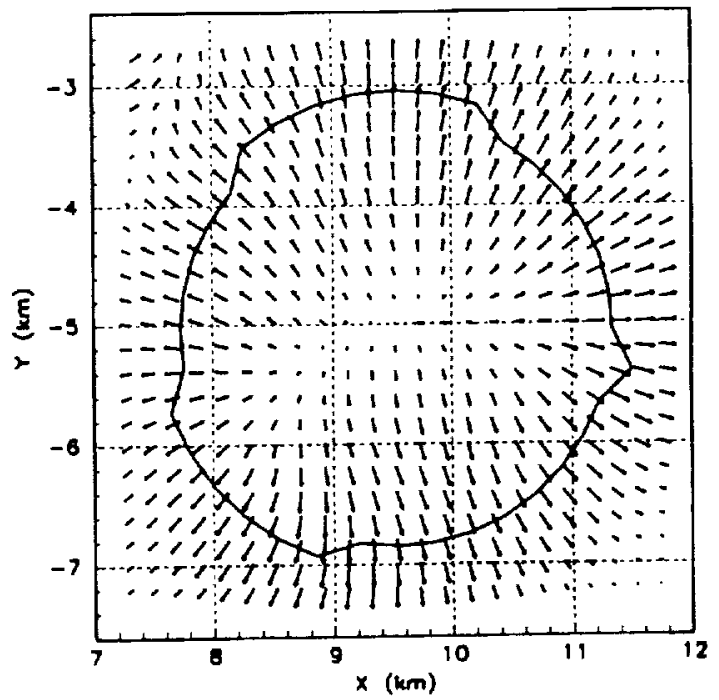

Figure 3: Microburst extent polygon. Horizontal windfield with outflow extent polygon superimposed.

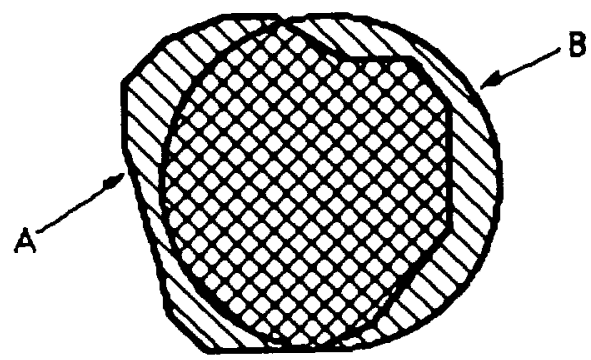

Figure 4: Extent Figure-of-Merit. Pictorial representation of Eqn. 22. The cross-hatched area is $A \cap B$ and the sum of the cross-hatched and striped areas is $A \cup B$. Note that the model "polygon" B is a circle for the single. microburst axisymmetric $\mathrm{OBV}$ model. 
Microburst intensity was defined in terms of " $F$. factor", proposed by researchers at NASA Langley Research Center, which is based on the impacl of a microburst windfield on the total energy (kinetic plus potential) of the aircraft. ${ }^{5}$ It is a measure of the loss of potential rate-of-climb (or loss of effective thrust-10weight ratio) due to the immediate windfield. It is dependent on the time rate of change in the aircraft frame of the tailwind velocity, the vertical wind velocity, and the aircraft airspeed. Positive values of $F$ indicate a performance-decreasing siluation, and negative values indicate a performance-increasing situation. As typical transport-category aircraft in landing or takeoff configuration have excess thrust-to-weight ratios between 0.1 and 0.15 , an encounter with an F-factor in excess of that value would compel the aircraft to descend and is therefore hazardous.

$$
F=\frac{\dot{W}_{s}}{g}-\frac{W_{h}}{V}
$$

$F$, a point measurement. needs to be averaged over a distance to give a useful indication of aircraft hazard. Past work has determined that $F$ averaged over $1 \mathrm{~km}$ of the aircraft flight path yields a good hazard estimate. ${ }^{7}$ For evaluation purposes. however, it is desirable to assign a single hazard number to a microburst rather than one for each possible flight path through it. Therefore. for this work. the hazard number was defined as follows: (1) compute $1 \mathrm{~km}$ average $F$-factors for a large number of parallel constant-altitude paths through the microburst. (2) average the resulting values $500 \mathrm{~m}$ laterally across flight paths, and (3) pick the largest averaged F-factor as the hazard value.

This value depends strongly on the direction of the flight paths along which $F$ is evaluated. In the simulation results presented below, averaged F-factors will be

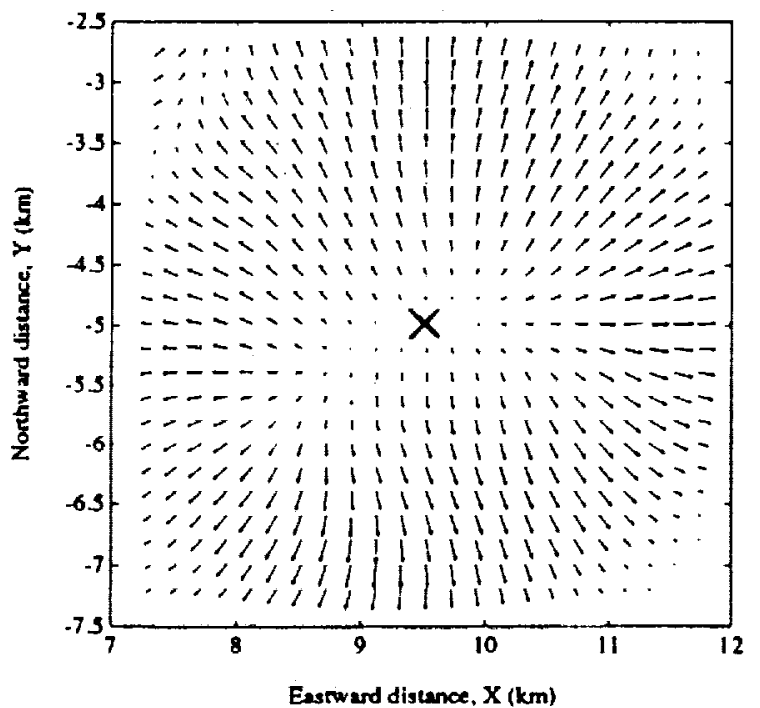

presented for either eastbound or nonhbound flight paths. In addition. $F$ depends on altitude, and results will therefore be presented for several altitudes. For alerting purposes, however, it would be necessary to assign a single intensity value to a detected microburst, for example the largest value (over all directions) below a specified maximum altitude.

\section{Simulated Microburst True Windfield}

The windfield data used to evaluate the estimation algorithm was generated by the Terminal Area Simulation System (TASS) 21 It is a highly detailed computational simulation of a complex multiple microburst event which occurred at Denver-Stapleton airport on July II, 1988. This event caused one near accident and a total of five aircraft to make missed approaches. 22 Windfield data from five times during this event was available, with a horizontal spacing of $200 \mathrm{~m}$ and a vertical spacing of approximately $80 \mathrm{~m}$. For the following analyses the largest microburst in the event was selected (Figure 5). The horizontal windfield has a classic microburst outflow pattern. However, the vertical wind contours show some complex structure as indicated by two separate regions of high downdraft, neither of which correspond to the apparent horizontal windfield center (marked with an X). This rather complex event was chosen to test the estimation algorithm in a challenging but realistic situation.

\section{OBV Model Best Fit}

The first step for algorithm evaluation was to determine the ability of the analytical model to match the important characteristics of a microburst windfield. namely the figures of merit defined above. This was done with a deterministic non-linear batch least-squares optimization algorithm, similar to that used in Ref. 2310 model microburst winds with vortex rings. The "truth"

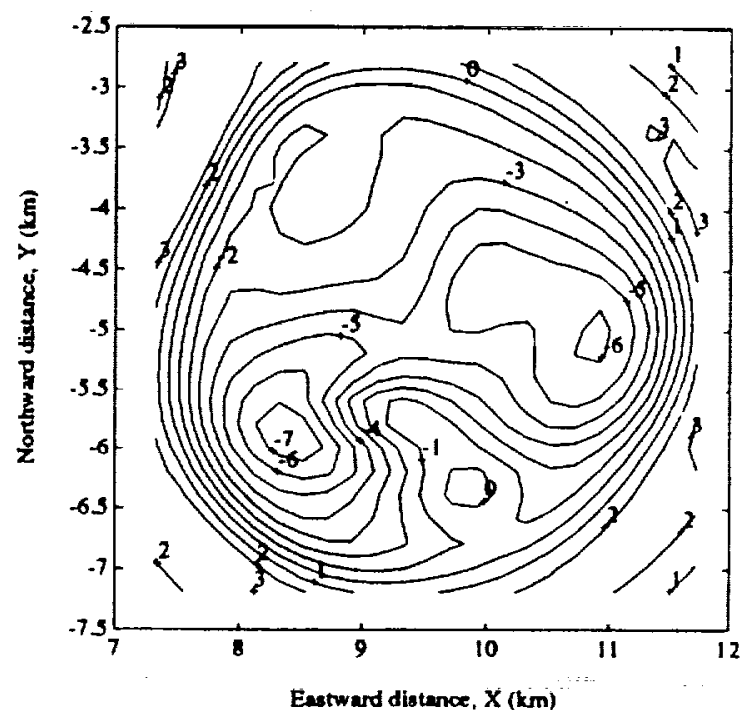

Figure 5. TASS-simulated windfield for 11 July 1988 microburst event at DEN. At left is a vector plot of horizontal winds: maximum velocity shown is $18.7 \mathrm{~m} / \mathrm{s}$. At right: vertical windspeed contours. Altitude shown is $177 \mathrm{~m}$ AGL. 
winds were taken from one time-step of the TASS model (shown in Figure 5). The data volume included three-axis winds at three altitudes with $200 \mathrm{~m}$ lateral spacing for a total of approximately 5000 data points. The optimization procedure was a constrained version of the standard Gauss-Newton method, 24 and found the model parameters which minimized the mean square wind error, J:

$$
J=\frac{1}{N} \sum_{j=l}^{N} e_{j}^{T}(p) e_{j}(p) ; \quad e(p)=V_{1 \text { ruth }}-V_{\text {mode }}(p)
$$

where $\mathrm{N}$ is the number of total data points and $\mathrm{V}$ is the vector of all wind points, including East, North, and vertical components at all $(x, y, z)$ locations. This procedure was done with a single-microburst model ( 9 parameters) and with a two-microburst model (14 parameters). The resulting parameters for the singlemicroburst case are given in Table 2 . Note that the approximate radius of this microburst is 1700 meters, and the maximum outflow speed is approximately $18 \mathrm{~m} / \mathrm{s}$. The ambient wind magnitude is small in this case.

Table 2: Single-microburst least-squares parameter fit results

\begin{tabular}{|l|c|l|c|}
\hline $\mathrm{x}_{0}(\mathrm{~m})$ & 9528 & $\mathrm{U}_{0}(\mathrm{~m} / \mathrm{s})$ & 0.9 \\
\hline $\mathrm{y}_{0}(\mathrm{~m})$ & -5047 & $\mathrm{U}_{\mathrm{h}}(\mathrm{m} / \mathrm{s} / \mathrm{m})$ & -0.001 \\
\hline $\mathrm{U}_{\mathrm{m}}(\mathrm{m} / \mathrm{s})$ & 17.8 & $\mathrm{~V}_{0}(\mathrm{~m} / \mathrm{s})$ & 0.5 \\
\hline $\mathrm{R}_{\mathrm{p}}(\mathrm{m})$ & 1717 & $\mathrm{~V}_{\mathrm{h}}(\mathrm{m} / \mathrm{s} / \mathrm{m})$ & -0.002 \\
\hline $\mathrm{Z}_{\mathrm{m}}(\mathrm{m})$ & 68.2 & & \\
\hline
\end{tabular}

The single-microburst fit produced an extent figure of merit of 0.92 . The two-microburst fit result was slightly lower, at 0.85 . As seen in Figure 5, this microburst was fairly axisymmetric in extent, so these good results are not surprising. However, plots of area-averaged F-factor looking Eastward and Northward for three altitudes (Figure 5) reveal that the microburst is not symmetric in intensity. As indicated by the "TASS windfield" points in Figure 6, the F-factors are larger when looking northward through the microburst than when looking eastward. This is due to the vertical wind distribution (Figure 5 . righthand plot) which has multiple regions of high vertical windspeeds. For this reason, the singlemicroburst fit produces a single broad region of somewhat weak vertical winds in an attempt to globally match the windfield, and the result is that intensity is underestimated in both directions. The two-microburst fit, on the other hand, succeeds in matching the vertical windfield well and duplicates the intensity of the TASS windfield well in both directions.

For alerting purposes, both model windfields adequately represent the actual extent; however, the singlemicroburst model underestimates the intensity somewhat. The results of previous work, however, indicale that highly accurate intensity estimates are not critical for alert generation. 9 Based on these results, and similar results obtained using TASS windfields from another microburst
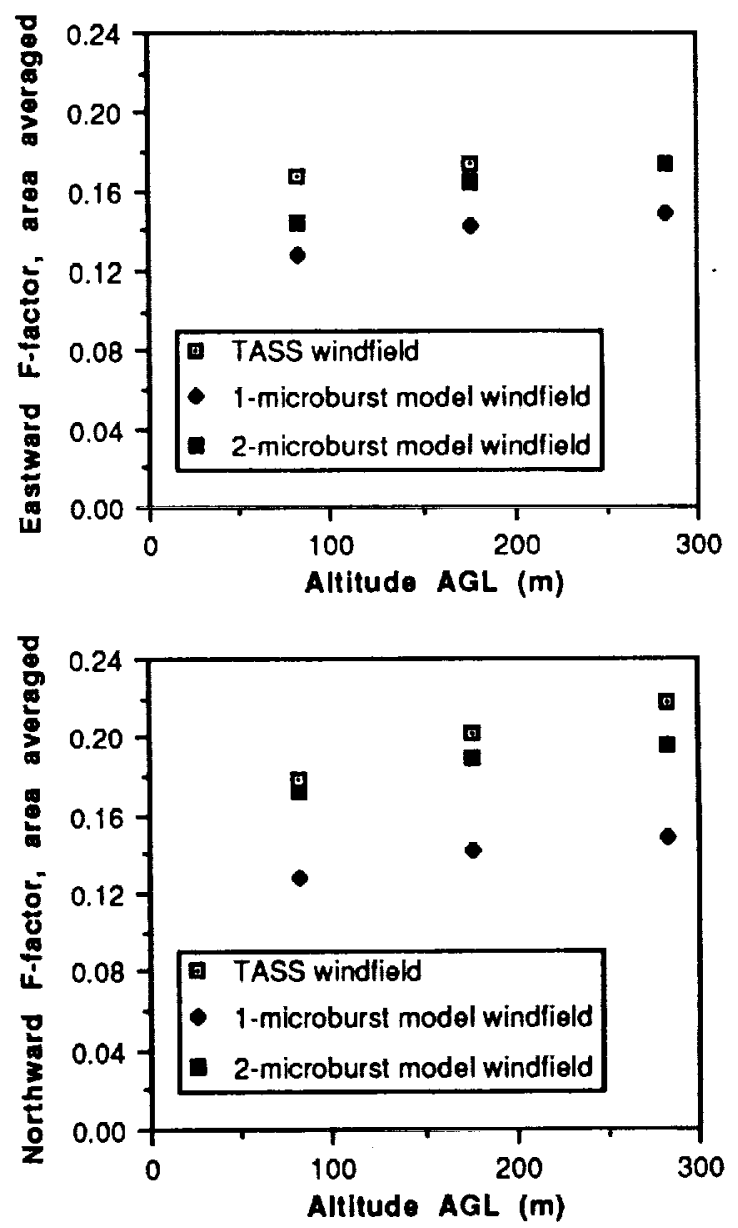

Figure 6: Microburst intensity matching performance for least-squares fit

event, the model was judged to be acceptable for estimation purposes.

\section{Iterated EKF Update Simulation}

The next step in algorithm evaluation was to determine if the iterated EKF update procedure was capable of taking sensor data (as modeled by small subsets of the entire windfield) and producing reasonable extent and intensity estimates. The TASS simulated winds were again considered to be the "truth" winds, and simulated sensor data subsets were taken from them. Assuming that the windfield was frozen in time (or alternatively, no time has lapsed between measurement sets), different combinations of sensor data were used sequentially to update the current estimate. Three sensors were considered in this way: (1) TDWR data, (2) winds measured from the aircraft, using inertial and air data measurements (referred to henceforth as INS data), and (3) airbome Doppler radar (ABDR) data.

For TDWR and ABDR data, it was assumed that the sensor was far enough from the microburst that radial 
wind measurements could be considered parallel to each other, and that the antenna tilt angle was horizontal so that all data was taken at the same altitude. For example, for an eastward-looking radar, the U-components of the TASS windfield at a single altitude became the working data set. TDWR measurements were taken at an altitude of $82 \mathrm{~m}$ AGL (the lowest TASS data altilude) for both eastward- and northward-looking cases, and ABDR measurements were taken from $177 \mathrm{~m}$ and $283 \mathrm{~m} \mathrm{AGL}$ TASS data. In all cases, gaussian zero-mean white noise with a standard deviation of $1 \mathrm{~m} / \mathrm{s}$ was added to the "truth" data to simulate measurement noise based on TDWR accuracy specifications. ${ }^{25}$ All radar data sets were taken at $400 \mathrm{~m}$ range and azimuth resolution; this is poorer than the resolution of operational radars, but reduced the computation time required to run the simulations.

Aircraft winds (INS data sets) consisted of 3 component winds along a straight flight path at constant altitude. Four $200 \mathrm{~m}$ resolution INS data sets were defined, including eastward and northward flight paths at $177 \mathrm{~m}$ and $283 \mathrm{~m}$ AGL. All paths passed through the approximate center of the windfield, as marked in Figure 5. The measurement noise standard deviation used for aircraft wind measurements was $1.4 \mathrm{~m} / \mathrm{s}$.

For simulation purposes, it was assumed that TDWR made the initial microburst detection. Therefore, the first step was to initialize the filter as previously described, and then apply the iterated EKF update to incorporate the TDWR measurement. The resultant parameter estimate and error covariance were saved. Then the estimate was updated by incorporating either an INS data set or an ABDR data set, starting with the saved parameter estimate and covariance matrix. Twelve tolal sensor fusion cases were tested with both one-microburst and two-microburst versions of the filter.

\section{Single-Microburst Filier}

For all cases tested, the iteration procedure used in the update converged in 3 to 5 iterations. Results for four representative cases are presented here:

(1) Initialization only: Eastward-looking TDWR measurements alone (denoted TDWR-E)

(2) The results of (1) were updated using a sequence of eastbound aircraft-measured winds taken at an altitude of $177 \mathrm{~m}$ AGL (denoted INS-E)

(3) The results of (1) were updated using a sequence of northbound aircraft-measured winds taken at an altitude of $177 \mathrm{~m}$ AGL (denoted INS-N)

(4) The results of (1) were updated using northwardlooking airbome Doppler radar data at $177 \mathrm{~m}$ AGL (denoted ABDR-N)

The extent results are again good (Table 3), and illustrate the effect of fusing data from sensors with different measurement geometries. The extent figure-ofmerit for TDWR-East is $\mathbf{0 . 8 5}$, and does not improve when
Table 3: Extent figures-of-merit: 1-microburst lime-invariant data fusion.

\begin{tabular}{|cccc|}
\hline $\begin{array}{c}\text { TDWR-E } \\
\text { alone }\end{array}$ & $\begin{array}{c}\text { TDWR-E } \\
+ \text { INS-E }\end{array}$ & $\begin{array}{c}\text { TDWR-E } \\
+ \text { INS-N }\end{array}$ & $\begin{array}{c}\text { TDWR-E } \\
+ \text { ABDR-N }\end{array}$ \\
0.853 & 0.853 & 0.911 & 0.917 \\
\hline
\end{tabular}

an eastward path of INS data is incorporated. However. when northbound INS data or northward-looking airborne radar data is incorporated, the extent figure-of-merit increases to the .91 to .92 range. Since the microburst is not exactly symmetric in extent (it is slightly larger in the north-south direction), incorporation of northward-looking data increases the radius parameter in the OBV model to cover more area. This is equal to the performance achieved by the least-squares fit computation.

The effect of multi-directional data is also visible in the intensity results (Figure 7). As with the least-squares results. it is clear that the single-microburst model cannot match intensity with the complex windfield of this microburst. The TDWR-alone result is low, and incorporating an eastbound path of INS data actually lowers the estimate; this is because the path does not cross both regions of high vertical windspeed.

Incorporating a northbound path of INS data or the ABDR data improves the estimate significantly at the higher altitudes, from which the INS and ABDR data are taken.

\section{Two-Microburst Filter}

The two-microburst version of the filter involved significantly more computation, since in general more iterations were required than for the single-microburst filter. Also, some cases did not converge consistently and required adjustment of the initial parameters. However. when the two-microburst filter did converge, the results were good. Extent figures-of-merit were between 0.85 and 0.90 for all cases. Figure 8 shows east ward intensity values for the algorithm applied to three cases:

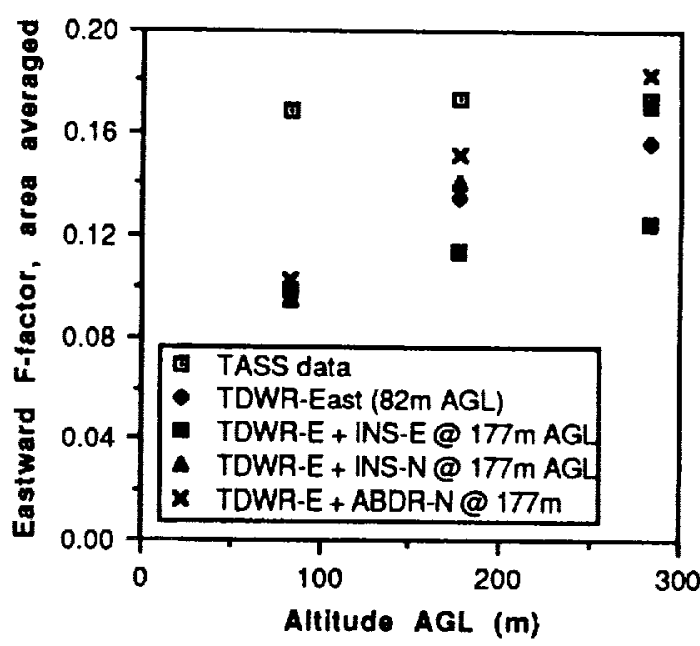

Figure 7: Intensity estimation - I-microburst time-Invariant data fusion 


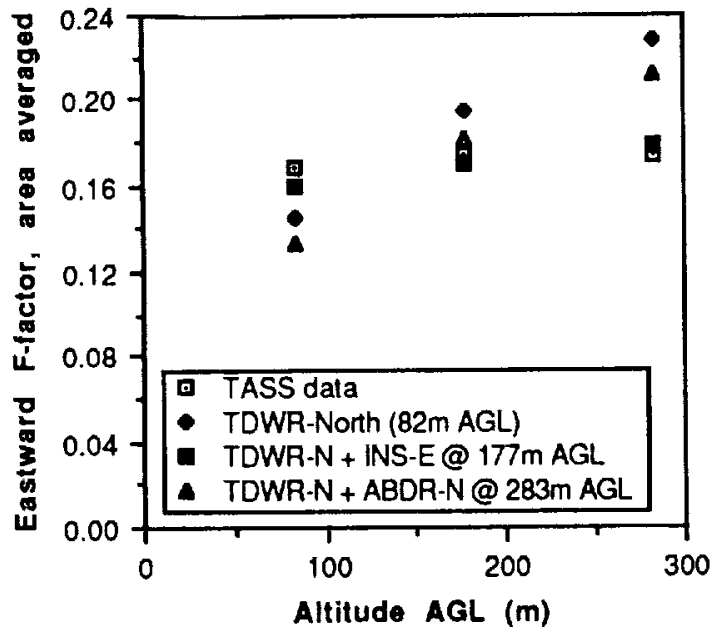

Figure 8: Intensity estimation - 2-microburst time-invariant data fusion

(1) northward-looking TDWR data alone (TDWR-N)

(2) TDWR-N updated with eastward aircraft-measured winds taken at $177 \mathrm{~m}$ AGL (INS-E)

(3) TDWR-N fused northward-looking airborne radar data at $283 \mathrm{~m}$ AGL (ABDR-N).

Although the TDWR is looking north and the intensity values shown are for eastbound paths, the results for TDWR data alone are fairly good. There is some overestimation at high altitudes. Inclusion of ABDR data with the same look angle as the TDWR (northward) improves the results slightly. As expected, inclusion of the eastbound INS data provides a second measurement direction and produces the best intensity estimates.

\section{Full Iterated EKF Simulation}

The third part of the algorithm evaluation was to include the microburst dynamic model (the propagation part of the filter) and apply the technique to time-varying data. For this analysis, data was taken from three different times in the evolution of the $7 / 11 / 88$ microburst event. The three data sets were spaced two minutes apart, where the middle data set corresponds to the time-invariant data set used in the previous section and corresponds to the time at which the microburst was strongest.

The time spacing for this data, was larger than desired, since TDWR data is updated at 1 min intervals and airborne radar data would be available even more frequently. However, it was still possible to construct illustrative examples. The following three sample cases assume that initial detection is made with northwardlooking TDWR. Two minutes later, three different events are postulated:
Case 1 A sequence of eastbound aircraft-measured winds is downlinked to the ground and incorporated along with a second sel of TDWR data

Case 2. An aircraft traveling northbound receives the previous TDWR estimate and updates using an airborne Doppler radar

Case 3. An aircraft traveling eastbound receives the previous TDWR estimate and updates using an airborne Doppler radar

At the third time step ( +4 minutes), the parameter set is passed to the ground and an update is done using another set of TDWR data. The data sets were derived in the same way as for the time-invariant cases, and the estimate and error covariance were propagated between measurements as described above. In all cases, the singlemicroburst form of the filter was used.

The extent figures-of-merit (Figure 9) are fairly good $(>0.82)$ through the first two times, but are slightly lower (0.76) in the third time step. This is due to the distorting effect of an adjacent, weaker microburst on the shape of the primary microburst. The axisymmetric model used in the filter has difficulty representing this

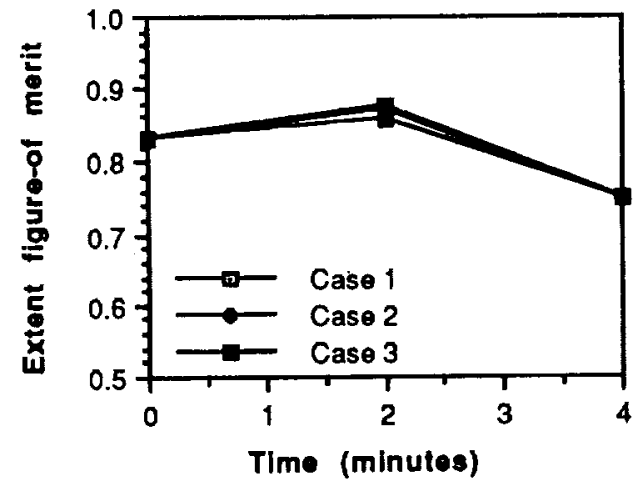

Figure 9: Extent figures-of-merit: timevarying cases

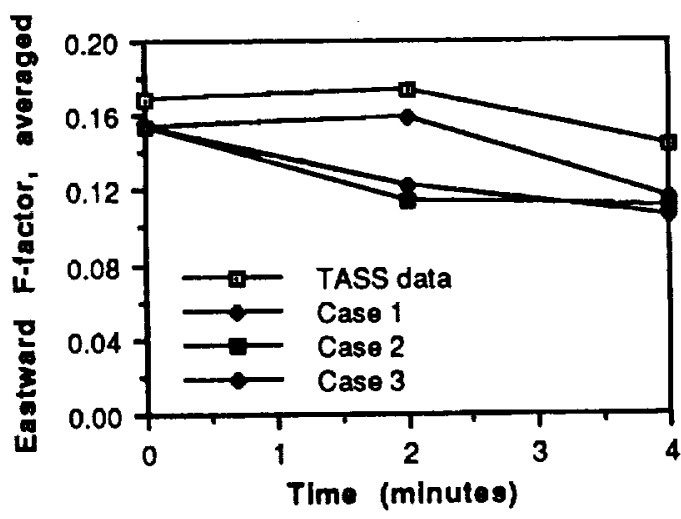

Figure 10: Eastbound Intensity estimates time-varying cases 
situation. There is little difference between the three sample cases.

The intensity results (Figure 10) are similar to those from the single-microburst time-invariant runs, in that all of the estimates are low. As evident from the "TASS data" curve, the actual microburst increases in strength in the first two minute span and then decreases in the last two minutes. Only in scenario 3, in which northbound TDWR measurements were combined with eastbound ABDR measurements, was the filter able to follow this trend. The low estimates are most likely due to difficulties matching this complex microburst with the single-microburst filter. However, the intensity results were some what sensitive to the choice of process noise strength, which indicates a need for further study of microburst time dynamics.

\section{Discussion}

The simulations demonstrate the potential usefulness of this technique. particularly for estimating the size and position of the microburst hazard region. Several other characteristics of the algorithm were also observed during the simulation runs, although it should be noted that the use of computational data for a single historical microburst event limits the scope of the conclusions that can be drawn. Further simulation work is planned, using data from actual field measurements.

The single-microburst algorithm appeared to be numerically robust. Errors in initial conditions and reasonable variations in choice of filter parameters did not produce filter instability in either the time-invariant or the time-varying simulations. The two-microburst form, however, was numerically sensitive. In several cases the filter diverged during the update iteration, and choice of parameters such as the initial covariance matrix appeared to have a large impact on the convergence properties of the filter. In cases where convergence was reached the results tended to be dependent on the actual windfield shape. When the windfield had two clear downdraft centers, the convergence was steady and the results for both intensity and extent were good. In cases where there was only one region of high downdraft (such as the first time-step of TASS model data) then the two sets of microburst parameters either became coincident, or one microburst became very weak. This mismodeling problem was also apparent in the diagonal elements of the covariance matrix; the covariance elements corresponding to the unnecessary microburst parameters grew very large. Possible solutions to this problem include more intelligent initialization based on recognized windfield features, or running multiple filters of different types in parallel. In any case, the improved estimation possible from the two-microburst filter must be weighed against the associated numerical difficulties.

Aside from numerical robustness and algorithm tuning issues, there are other implementation issues to be considered. The computational requirements of the filter need to be assessed with respect to available computational resources. Computational load can be decreased by thinning large data sets, at the expense of estimation accuracy. Also, datalink bandwidth needs to be considered. A model parameter list of 9 elements, for example, has an associated 81 element covariance matrix (of which only 45 are unique). It is likely that the entire covarianice matrix is not necessary to initialize the next update step, and that some elements could be omitted without loss of performance.

Although the algorithm has been presented in the context of multi-sensor data fusion, it does not require multiple sensors. Benefits would still be gained if it were used with a single sensor due to the additional information contained in the analytical model (correct fluid dynamics, empirical data). Also, the algorithm could be adapted to other fluid dynamic phenomena which can be represented by simple analytical models.

\section{Conclusion}

A recursive model-based data fusion algorithm for multi-sensor microburst hazard assessment was presented. A simple analytical microburst model is used to approximate the actual windfield, and a "best" set of model parameters are estimaied from measured winds using an extended Kalman filtering technique. The resulting parameter estimate and associated error covariance encapsulate the current state of knowledge about the actual windfield, and can be used to compute estimates of microburst position, extent, and intensity for alent generation. Microburst state dynamics and process noise parameters for the filter were chosen based on statistical data from microburst field studies.

Simulated measurements for three types of sensors were derived from a time-varying computational model of a historical microburst event. Two forms of the algorithm were then tested, one using a single-microburst model and one using a two-microburst model. It was found through both time-invariant and time-varying simulations that both forms of the algorithm were able to estimate the position and extent of the simulated microburst well. The two-microburst model produced better intensity estimales, but suffered from numerical robustness problems. These preliminary results are promising, and further work is planned including simulations using field measurements and study of feasibility issues such as computational requirements.

\section{Acknowledgements}

The authors would like to thank Steven Campbell of MIT Lincoln Laboratory and David Hinton and Fred Proctor of the National Aeronautics and Space Administration (NASA), Flight Management Division, Langley Research Center, for their input, advice, and support. This work was supported by NASA and MIT. Lincoln Laboratory under Air Force Contract No. F19628$90-\mathrm{C}-0002$, and the Federal Aviation Administration and NASA under grant NGL-22-009-640. 


\section{References}

1. National Research Council, Low Altitude Wind Shear and lis Hazard to Aviation, National Academy Press, 1983.

2. Evans, James E., "Status of the Terminal Doppler Weather Radar One Year Before Deployment," Ath International Conference on Aviation Weather Systems, Paris, France, June 24-26. 1991. American Meteorological Society. Boston, MA.

3. Campbell, Steven D., Merritt, Mark W., and DiStefano, John T., "Microburst Recognition Performance of TDWR Operational Testbed," $4 t h$ International Conference on Aviation Weather Systems. Anaheim, CA, January 30 - February 3. 1989. American Meteorological Society, Boston, MA.

4. Wilson, F. Wesley. Jr., and Gramzow, Richard H., "The Redesigned Low Level Wind Shear Alert System," 4ih International Conference on Aviation Weather Systems, Paris, France, June 24-26, 1991 , American Meteorological Society, Boston, MA.

5. Bowles, Roland L., "Reducing Windshear Risk Through Airborne Systems Technology," 17th Congress of the International Council of the Aeronautical Sciences, Stockholm, Sweden, September 9-14, 1990.

6. Orlando, V. A., and Drouilhet, P. R., Mode S Beacon System: Functional Description, DOT/FAA/PM-86/19, Lincoln Laboratory, Massachusetts Institute of Technology, Lexington, MA, August 1986.

7. Wanke, C., and Hansman, R. J., "Alert Generation and Cockpit Presentation for an Integrated Microburst Alerting System," AIAA Paper 91-0260, 29th Aerospace Sciences Meeting, Reno, NV, USA, January 7-10, 1991.

8. Wanke, C., Chandra, D., Hansman, R. J., and Bussolari, S. R., "A Comparison of Voice and Datalink for ATC Amendments and Hazardous Wind Shear Alerts," 4th Intemational Symposium on Aviation and Space Safety, Toulouse, France, 20-22 November 1990.

9. Wanke, C., and Hansman, R. J., "Experimental Evaluation of Candidate Graphical Microburst Alert Displays," AIAA Paper 92-0292, 30th Aerospace Sciences Meeting, Reno, NV, USA, January 6-9. 1992.

10. Comman, Larry B., and Mahoney, William P. III, "Integration of the TDWR and LLWAS Wind Shear Detection Systems," 4th International Conference on Aviation Weather Systems, Paris, France, June 2426, 1991. American Meteorological Society, Boston, MA.
11. Stratton, D. Alexander, and Stengel, Robert F., "Probabilistic Reasoning for Intelligent Wind Shear Avoidance," Journal of Guidance, Control, and Dynamics, Vol. 15, No. 1, Jan.-Feb. 1992.

12. Oseguera, Rosa M., and Bowles, Roland L., "A Simple Analytic 3-Dimensional Downburst Model Based on Boundary Layer Stagnation Flow," NASA Technical Memorandum 100632, July 1988.

13. Vicroy, Dan D., "A Simple, Analytical, Axisymmetric Microburst Model for Downdraft Estimation," NASA Technical Memorandum 104053, February 1991.

14. Hallowell, Robert G., "Aspect Angle Dependence of Outflow Strength in Denver Microbursts: Spatial and Temporal Variations," 16th Conference on Severe Local Storms, Kananaskis Park, Alberta, Canada, October 22-26, 1990, American Meteorological Society, Boston, MA.

15. Gelb, A., ed., Applied Optimal Estimation, MIT Press, Cambridge, MA, 1974.

16. Wilson, J. W., Roberts, R. D., Kessinger, C., and McCarthy, J., "Microburst Wind Structure and Evaluation of Doppler Radar for Airport Wind Shear Detection," Journal of Climate and Applied Meteorology, Vol. 29, June 1984.

17. Hjelmfelt, Mark R., "Structure and Life Cycle of Microburst Outflows Observed in Colorado," Journal of Applied Meteorology, Vol. 27, August 1988.

18. Jazwinski, A. H., Stochastic Processes and Fillering Theory, Academic Press, New York, 1970.

19. Wishner, R. P., Tabaczynski, J. A., and Athans, M., "A Comparison of Three Non-Linear Filters," Automatica, Vol. 5, 1969.

20. Biron, P. J., and Isaminger, M. A., High Resolution Microburst Outflow Vertical Profile Data from Huntsville, Alabama, and Denver, Colorado, Project Report ATC-163, Lincoln Laboratory, Massachusetts Institute of Technology, Lexington, MA., April 1991.

21. Proctor, Fred H., The Terminal Area Simulation System-Volume I: Theoretical Formulation; Volume II: Verification Cases, NASA CR-4046 and CR-4047, DOT/FAA/PM-86/50, 1987.

22. Schlickenmaier, Herbert W., Windshear Case Study: Denver, Colorado, July 11,1988 . Federal Aviation Administration, DOT/FAADS-89/19, 1989.

23. Schultz, Thomas A., "Multiple Vortex Ring Model of the DFW Microburst," Journal of Aircraft. Vol. 17, No. 2, Feb. 1990. 
24. Scales, L. E., Introduction to Non-Linear

Optimization, Springer-Verlag, New York, 1985.

25. Personal Communication with Steven Campbell,

Lincoln Laboratory, June 1992.

\section{Apoendix A: Oseguera-Bowles-Vicroy}

\section{Microburst Model Equations}

The $U, V$, and $W$ wind components are functions of position $(x, y, z)$ and the model parameters:

$$
\begin{aligned}
& \mathrm{u}=\frac{\lambda \tilde{x}}{2}\left[\mathrm{e}^{c_{1}\left(z / z_{m}\right)}-\mathrm{e}^{c_{2}\left(z / z_{\mathrm{m}}\right)}\right] \mathrm{e}\left[\frac{2 \cdot\left(\tilde{x}^{2}+\tilde{y}^{2}\right) /_{\mathrm{rp}_{\mathrm{p}} \alpha \alpha}}{2 \alpha}\right] \\
& v=\frac{\lambda \tilde{y}}{2}\left[\mathrm{e}^{\mathrm{C}_{1}\left(\mathrm{z} / z_{\mathrm{m}}\right)}-\mathrm{e}^{\mathrm{C}_{2}\left(\mathrm{z} / \mathrm{z}_{\mathrm{m}}\right)}\right] \mathrm{e}\left[\frac{2 \cdot\left(\tilde{\mathrm{x}}^{2}+\tilde{y}^{2}\right)^{\alpha} / \mathrm{r}_{\mathrm{p}} 2 \alpha}{2 \alpha}\right] \\
& w=-\lambda\left\{\frac{z_{m}}{C_{1}}\left[e^{C_{1}\left(z / z_{m}\right)}-1\right]-\frac{z_{m}}{C_{2}}\left[e^{C_{2}\left(z / z_{m}\right)}-1\right]\right\} \\
& \times\left[1-\frac{\left(\tilde{x}^{2}+\tilde{y}^{2}\right)^{\alpha}}{2 r_{p}^{2 \alpha}}\right] e^{\left[\frac{2 \cdot\left(\tilde{x}^{2}+\tilde{y}^{2}\right)^{\alpha} / r_{p} 2 \alpha}{2 \alpha}\right]}
\end{aligned}
$$

where the position offsets are given by

$\tilde{\mathrm{x}}=\mathrm{x}-\mathrm{x}_{0}$

$\tilde{y}=y-y_{0}$

and the radial scale factor $\lambda$ is:

$$
\lambda=\frac{2 u_{m}}{r_{p}\left(e^{c_{1}}-e^{c_{2}}\right) e^{(1 / 2 \alpha)}}
$$

$C_{1}$ and $C_{2}$ are empirically adjusted constants with the following values:

$$
\begin{aligned}
& C_{1}=-0.15 \\
& C_{2}=-3.2175
\end{aligned}
$$

and $\alpha$ is a shaping parameter which was set to 2.0 for the work presented here. 
$0^{11}$

\section{OHIO UNIVERSITY}


